\title{
Printed electronic on flexible and glass substrates
}

\author{
Konrad Futera, ${ }^{* 1,2}$ and Małgorzata Jakubowska ${ }^{2}$ \\ ${ }^{1}$ Instytut Tele-i Radiotechniczny, ul. Ratuszowa 11, 03-450 Warszawa, \\ ${ }^{2}$ Institute of Metrology and Biomedical Engineering, Warsaw University of Technology \\ ul. Św. Andrzeja Boboli 8, 02-525 Warszawa
}

Received June 21, 2010; accepted June 23, 2010; published June 30, 2010

\begin{abstract}
Organic electronics is a platform technology that enables multiple applications based on organic electronics but varied in specifications. Organic electronics is based on the combination of new materials and cost-effective, large area production processes that provide new fields of application. Being characterized by size, weight, flexibility and environmental friendliness, organic electronics enables low cost production of numerous electrical components and provides for such promising fields of application as: intelligent packaging, low cost RFID, flexible solar cells, disposable diagnostic devices or games, and printed batteries [1]. The paper presents technology of manufacturing electronic elements on flexible and glass substrates. Fabricated structures were characterized form the point of shape, surface and geometry. A variety of substrates were investigated, within some, low cost, non-specialized substrate, designed for other purposes than organic electronics. Results of substrates influence on printing process are described in the present paper.
\end{abstract}

Organic electronics can be fabricated using well known printing techniques, out of which, roll-to-roll techniques are the most desirable. They require, however, expensive tools accompanied with, high cost test runs. Hence, they are inconvenient for research and development laboratory tests. For this reason, small companies and research institutes are more interested in InkJet technology, perfect for small scale production and prototyping. Inkjet printers do not require tools or process changes for fabricating new set of elements. They lower the cost of prototype fabrication by printing from graphic bitmap files straight to the substrates. This makes InkJet printing the leader of organic electronics fabrication technologies. For this reason, the investigation of the inkjet printing process is crucial for the fabrication of printing electronics issue.

In Tele- and Radio Research Institute investigations and tests have been made on self-built InkJet printer. The printer is based on a MicroDrop piezo-electric printing head, with a nozzle diameter of $50 \mu \mathrm{m}$, and drops volume from 35 to 55 pico litters. The $\mathrm{pH}$ of ink is to be in the 112 range, and the viscosity in a range of $1-15 \mathrm{cP}$ [2]. This allows to test a large variety of commercial inks and new inks produced by companies and other research institutes. The printer's schematic diagram is shown in Fig. 1.

\footnotetext{
*E-mail: Konrad.futera@itr.org.pl, k.futera@mchtr.pw.edu.pl
}

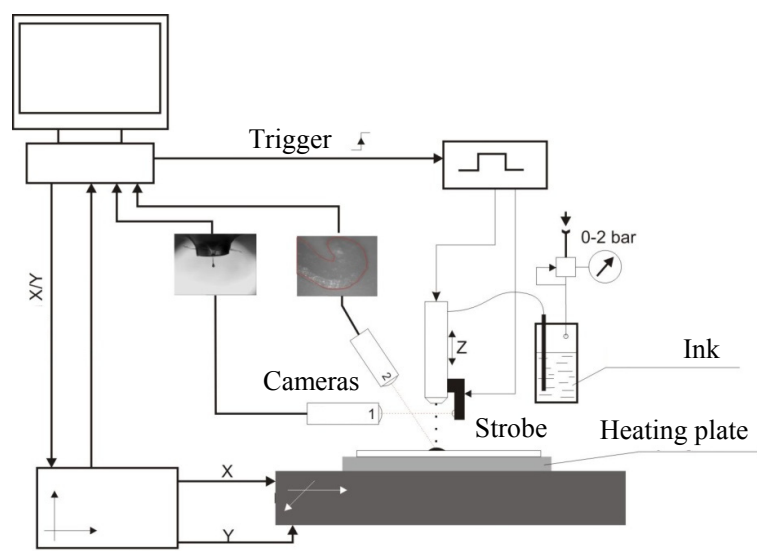

Fig. 1. InkJet printer schematics.

The printer is designed for investigating organic ink features, printing parameters, ink/print head and ink/substrate compatibility, and printing electronic elements as well. However, it is possible to use inorganic inks based on metal powders, such as nanosilver based inks. Printer with its control unit is shown in Fig. 2.

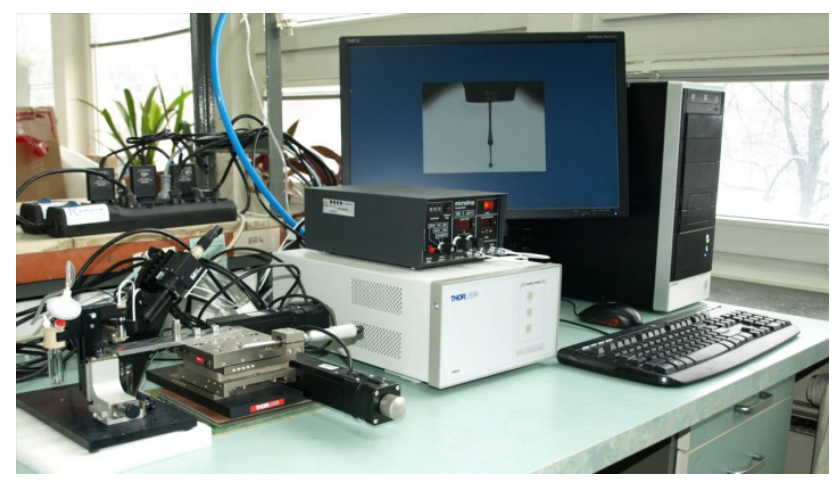

Fig. 2. ITR Inkjet printer.

Two CCD cameras are used to control the printing process. Camera one is used to control jetted drop shape, size and trajectory. The second camera controls drop deposition on a substrate and pattern quality. Drop size and shape can be changed by adjusting amplitude, delay and frequency of an electric control impulse. Control parameters must be adjusted and optimized for each ink 
because of their very different viscosity, in ranges of 10 $250 \mathrm{~V}$ and $2-100 \mu \mathrm{s}$.

In this work, the result of tests on PEDOT:PSS H.C.Stark based organic ink for inkjet printing is presented. Printer parameters were adjusted to get a single drop on demand, which is important for high quality inkjet printing. PEDEOT:PSS ink parameters were set to: $60 \mathrm{~V}$ voltage and $4 \mu \mathrm{s}$ length of print head piezoelectric impulse. Ink and substrate temperature was set to $25^{\circ} \mathrm{C}$. With these settings a single drop of PEDOT:PSS could be jetted. A single drop jetted on demand is shown in Fig. 3.

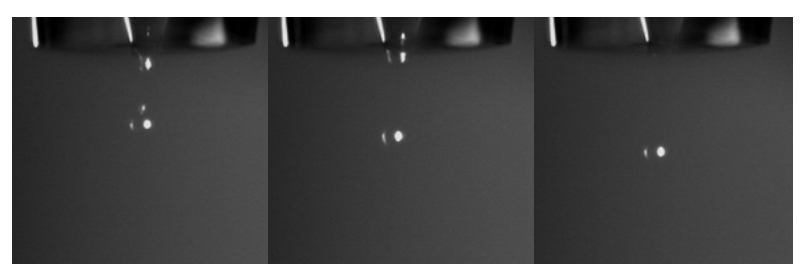

Fig. 3. Single drop of PEDTO:PSS.

Single drops were deposited on different substrates to evaluate ink/substrates compatibility. Tests substrates were glass, paper designed for organic electronic inkjet printing and regular photo paper.

Glass was chosen as a non absorptive, easy to prepare surface substrate. It is an ideal material for investigating surface phenomena in drop deposition. The results of printing PEDETO:PSS on glass are demonstrated in Fig.4.

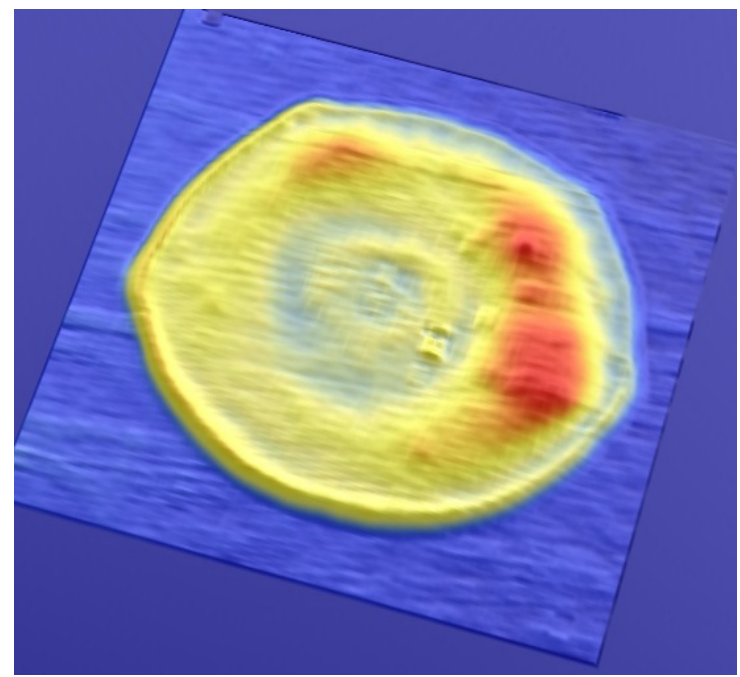

Fig. 4. PEDOT:PSS drop, printed on glass.

The profile measured on a laser profilemeter shows that the edges of the dot are much higher that the center (Fig. 6). This effect is the so-called "coffee ring" or "donut" effect.

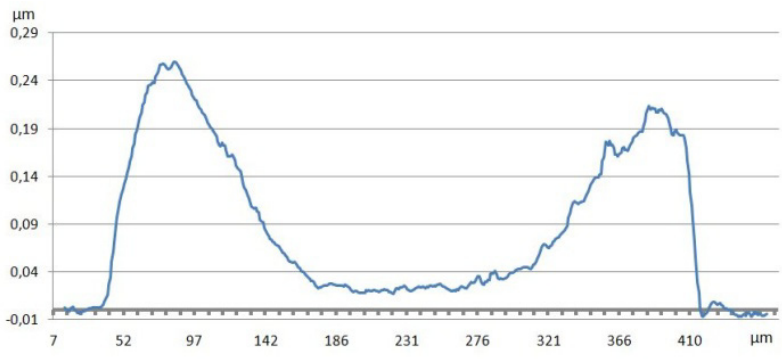

Fig. 5. PEDOT:PSS drop printed on a glass.

This effect was because of ink deposited on a substrate spreads forming a wave, with the solvent evaporating so fast that the wave is not able to go back and form regular shape. The methods of reducing the "coffee ring" effect were not an issue in these investigations. However, a higher temperature of the substrate caused faster solvent evaporation, making wave spreading harder and thus reducing "coffee ring" effect. Obtained dot diameter was $350 \mu \mathrm{m}$, and $253 \mathrm{~nm}$ of maximum height.

The next substrates used in investigation were Felix Schoeller smart papers. With polymer layer laminated on the surface, they were specially designed for printed organic electronics [3] (Fig. 6). This polymer layer reduces absorption and by using different polymers, the surface energy can be controlled.
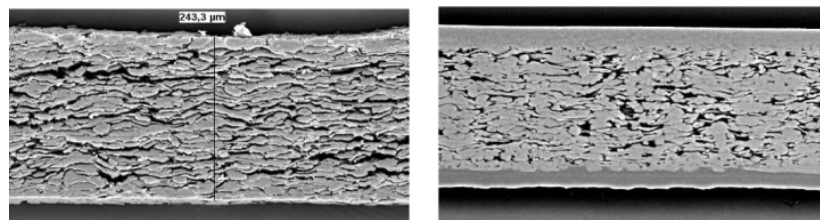

Fig. 6. Standard paper, and foil covered paper [4]

Printed dots on a paper substrate are shown in Fig. 7.

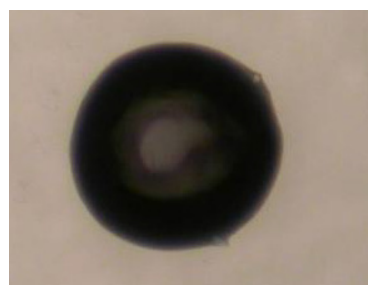

Fig. 7. PEDOT:PSS dot on Felix Schoeller paper.

The dot profile as shown in Fig. 8 is sferoidal. There is no "coffee ring" effect, and the suface is relatively smooth. Diameter of the dot is $62 \mu \mathrm{m}$ and height is $15 \mu \mathrm{m}$.

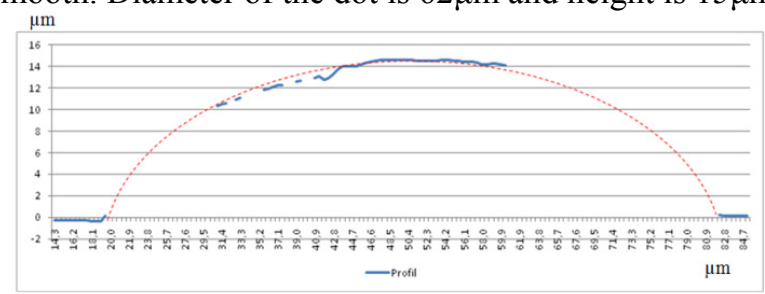

Fig. 8. PEDOT:PSS dot printed on a Felix Schoeller paper. 
Printing conductive lines on a glass and flexible substrate was the main issue of investigation. Every electronic element is built of lines made of materials with different features. Fabricating lines on a glass surface needs overcoming the "coffee ring" effect. One of the most popular methods to overcome this - i.e., printing dots in spacing less than the dot diameter, was tested. The results of PEDOT:PSS conductive line is shown in Fig. 9.

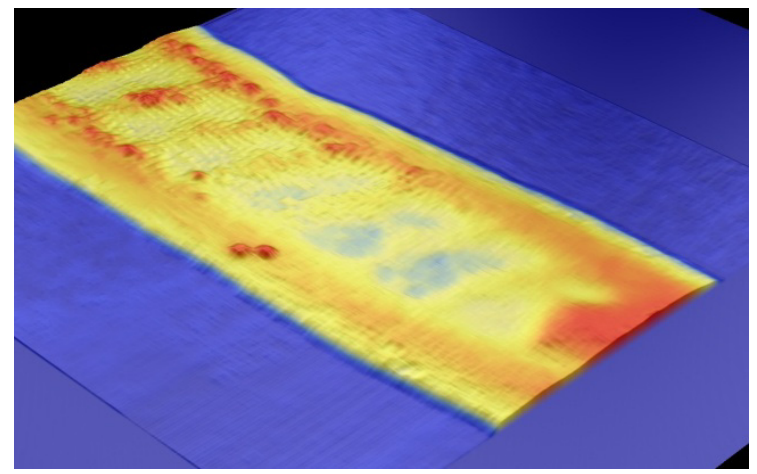

Fig. 9. Conductive line of PDEOT:PSS on glass.

Dots printed close to each other form a leader line, they should be printed close enough to form a smooth surface [4]. The width of the obtained line is a dot diameter - about $350 \mu \mathrm{m}$, and its height is up to $300 \mathrm{~nm}$.

The line printed on paper (Fig. 10) has smoother surface, and the line is continuous. However, printing on paper was more difficult. The electric charge on a surface can change the trajectory of a jetted drop. Removing the surface charge form paper is difficult. The charge can be also easily generated from air.

Printing on standard photo paper was unsuccessful, due to absorption, surface roughness, foil cover thickness which was different in random places. It occurred that standard photo paper is useless for ink jet printing electronics.

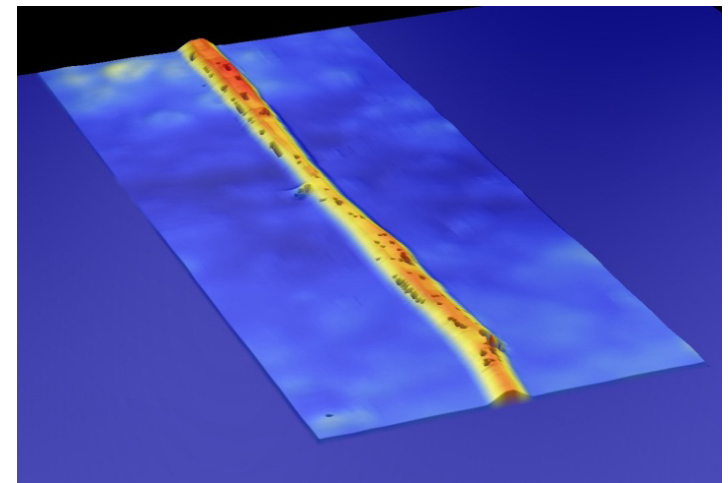

Fig 10. Conductive line printed on Felix Schoeller paper.

In conclusions, the paper presents first results of fabricating layers with the use of self constructed simple ink jet printer. For low cost and flexible electronic applications conductive, polymer tracks were successfully fabricated. Some aspects of compatibility of Ink/substrate was investigated. Suitable substrates materials and fabrication parameters, for printing PEDOT:PSS on flexible substrates has been chosen. The next step is to put numbers of tracks made from different materials into specific patterns, making it fully operating electronic device printed on this single machine.

\section{References}

[1] White Paper "OE-A Roadmap for Organic and Printed Electronics". Organic Electronic Association, 2008.

[2] MicorDrop datasheet: www.microdrop.de

[3] W. Schmidt, F. Schoeller, Service GmbH \& Co. KG, Paper Based Substrates for Printed Electronics, Large-area, Organic \& Printed Electronics - Convention (LOPE-C), June 23-25, 2009, Frankfurt, Germany.

[4] V. Subramanian, Printed Zinc Oxide Based Electronics: Materials, Devices and Outlook, Large-area, Organic \& Printed Electronics Convention (LOPE-C), June 23-25, 2009, Frankfurt, Germany. 